\title{
Hole-doped two-dimensional InSe for spintronic applications
}

\author{
K. Iordanidou ${ }^{1}$, M. Houssa ${ }^{2}$, J. Kioseoglou ${ }^{3}$, V. V. Afanas'ev ${ }^{2}$, A. Stesmans ${ }^{2}$, C. Persson ${ }^{1}$ \\ ${ }^{1}$ Centre for Materials Science and Nanotechnology, Department of Physics, University of Oslo, \\ P.O. Box 1048 Blindern, NO-0316 Oslo, Norway \\ ${ }^{2}$ Department of Physics and Astronomy, University of Leuven, B-3001 Leuven, Belgium \\ ${ }^{3}$ Department of Physics, Aristotle University of Thessaloniki, GR-54124 Thessaloniki, Greece
}

Corresponding authors:

konstantina.iordanidou@smn.uio.no

michel.houssa@kuleuven.be

\begin{abstract}
Using first-principles calculations based on density functional theory, we study the magnetic and electronic properties of hole-doped two-dimensional InSe. Our simulations reveal that although 2D InSe is intrinsically non-magnetic, a stable ferromagnetic phase appears for a wide range of hole densities. Interestingly, hole doping not only induces spontaneous magnetization but also half-metallicity, and hole-doped InSe, presenting one conducting and one insulating spin channel, could be highly promising for next generation spintronic nanodevices. The possibility to induce hole doping and a subsequent ferromagnetic order by intrinsic and extrinsic defects was also investigated. We found that In vacancy creates spin-polarized states close to the valence band and leads to a p-type behavior. Similar to In vacancies, group-V atoms replacing Se atoms
\end{abstract}


lead to a p-type behavior, potentially stabilizing a ferromagnetic order in 2D InSe.

Keywords: defects, ferromagnetism, half-metallicity, hole-doping, InSe

\section{Introduction}

Over the last decades, the miniaturization of field effect transistors (FETs) has yielded significant improvements in power efficiency. However, the conventional silicon technology is reaching its limits for ultra-scaled devices, both in terms of performance degradation and power dissipation [1]. Two-dimensional (2D) materials have been proposed as promising candidates to replace silicon as a channel material $[2,3,4,5,6,7]$. Although graphene is a fascinating material with superior charge carrier mobility [8], the lack of band gap is its Achilles' heel. Thus, ongoing research is focusing on other 2D systems, like group-III mono-chalcogenides (e.g. GaS, GaSe, and InSe) $[9,10,11,12,13,14,15,16,17,18]$.

InSe is a layered material with strong intralayer bonding and weak van der Waals interlayer bonding. Each monolayer consists of four hexagonal atomic planes with the stacking sequence of Se-In-In-Se. Similar to other layered materials, few-layer InSe can be mechanically exfoliated from the bulk crystal, using the adhesive tape method $[19,20]$. A solution-processable fabrication technique for ultrathin nanosheets has been reported. This technique results in nanosheets which are separated from each other by organic ligands, and yields crystal thicknesses which are hardly accessible by exfoliation techniques [21].

Recent experimental studies revealed that InSe is a promising candidate for high performance nanoelectronic devices in the post-Si era. The electron mobility of few-layer InSe encapsulated 
in hexagonal boron nitride, under an inert atmosphere, exceeds $10^{3}$ and $10^{4} \mathrm{~cm}^{2} \mathrm{~V}^{-1} \mathrm{~S}^{-1}$ at room and liquid-helium temperature, respectively [20]. In addition, the two-terminal room-temperature field effect mobility of InSe FET with PMMA coated on $\mathrm{Al}_{2} \mathrm{O}_{3}$ as dielectric layer, is found to reach $\sim 1000 \mathrm{~cm}^{2} \mathrm{~V}^{-1} \mathrm{~s}^{-1}$, which is comparable to that of strained silicon thin-film [22].In addition, the radiative recombination of photo-excited carriers bound at native donors and acceptors in exfoliated nanoflakes of nominally undoped $\gamma$-InSe was reported, and their binding energies were found to increase by decreasing the flake thickness [23].

Recently, InSe-based van der Waals heterojunctions were investigated. Padilha et al. proposed a p-n heterojunction composed by bilayer phosphorene and monolayer InSe where the $\mathrm{p}$-type/ntype doping levels can be tuned by an external electric field at the semiconductor/metal contact regions [24]. Also, 2D $\mathrm{n}-\mathrm{InSe} / \mathrm{p}-\mathrm{GeSe}(\mathrm{SnS})$ heterojunctions were proposed for future optoelectronic devices, due to their effective charge separation, relatively high carrier mobility, broad optical absorption spectrum, and high optical absorption intensity [25].

Owing to their promising applications in spintronics, diluted magnetic semiconductors (DMS) and diluted magnetic oxides (DMO) have triggered intense interest. Many efforts have been made to prepare DMS/DMO by doping conventional non-magnetic semiconductors/oxides with magnetic impurities. Interestingly, unexpected room-temperature ferromagnetism in the absence of magnetic impurities, has been additionally observed [26,27,28,29,30,31,32,33]. In the emerging field of spin-based nanoelectronics, group-III mono-chalcogenides may offer a great potential. In particular, spontaneous magnetization has been theoretically predicted in hole-doped $\mathrm{GaS}$ and GaSe monolayers [34,35]. Such magnetization arises from an exchange splitting of the electronic states at the valence band top, where the density of states exhibits a sharp Van Hove 
singularity, resulting in the so-called Stoner instability.

In this paper, using first principles calculations based on density functional theory, we studied the magnetic and electronic properties of hole-doped two-dimensional InSe. We found that although 2D InSe is intrinsically non-magnetic, a stable ferromagnetic phase appears for a wide range of hole densities. The possibility to induce hole doping and a subsequent ferromagnetic order by intrinsic and extrinsic defects was also investigated.

\section{Models and Computational Methods}

First principles calculations of InSe monolayers are performed using the spin polarized DFT, as implemented in the Vienna ab-initio simulation package (VASP) [36,37]. The electron-ion interaction is described by the projector augmented wave (PAW) method [38], and the generalized gradient approximation, developed by Perdew, Burke and Ernzerhof (PBE), is used

for the exchange correlation functional [39]. Long-range van der Waals corrections are applied, using the DFT-D3 Grimme method [40]. The atomic positions are optimized using the conjugate gradient method.

We constructed $5 \times 5$ single-layer supercells containing two In atomic planes sandwiched between two Se atomic planes, with $\sim 20.3 \AA$ x $20.3 \AA$ lateral dimensions and 100 atoms in total. In addition, $3 \times 3$ bilayer supercells with $\sim 12.2 \times 12.2 \AA$ lateral dimensions and 72 atoms in total were considered. Periodic boundary conditions were applied, and a vacuum layer larger than 15 $\AA$ was used in the out-of-plane direction, to eliminate interaction between periodic images. The kinetic energy cutoff was set at $500 \mathrm{eV}$ and the Brillouin zone was sampled by a $6 \times 6 \times 1$ k-point grid for the atomic relaxations. For the band structure calculations, we used a grid of 45 k-points 
in total, i.e., 15 k-points in each of $Г \mathrm{M}, \mathrm{MK}$, and $\mathrm{K} \Gamma$ directions. Note that for hole-doped monolayers (bilayers) a very dense grid of $16 \times 16 \times 1(30 \times 30 \times 1)$ k-points was considered, to get well-converged magnetic moments.

To obtain more accurate defect level positions with respect to the band edges, we additionally performed hybrid functional calculations, using the VASP code. The functional proposed by Heyd, Scuseria and Ernzerhof (HSE) was adopted [41] and the fraction of the Fock exchange was set at 0.25 . We note that for HSE calculations, smaller supercells containing 36 atoms were employed.

To evaluate the stability of the defective configurations and the persistence of ferromagnetism, we performed first-principles molecular dynamics simulations based on DFT. We used the canonical NVT ensemble at $\mathrm{T}=300 \mathrm{~K}$, and the equations of motions were integrated for $5 \mathrm{ps}$ with 1 fs time step.

\section{Results and Discussion}

3.1 Ferromagnetism and half-metallicity in hole-doped InSe

The relaxed atomic structure of InSe monolayer is shown in Fig.1a. For the optimized unit cell, the computed in plane lattice constant is $4.06 \AA$. The Se-In bond length, In-In bond length, and Se-In-In bond angle are found to be $2.68 \AA, 2.79 \AA$, and $118.8^{\circ}$, respectively, in excellent agreement with previously reported theoretical calculations $[42,43]$.

As shown in Fig.1b, single-layer InSe is a semiconductor with a computed indirect band gap of $\mathrm{E}_{\mathrm{g}}=1.51 \mathrm{eV}$, in good agreement with previously reported DFT-PBE calculations [42]. The valence band (VB) top lies between $\mathrm{K}$ and $\Gamma$ points, whereas the conduction band $(\mathrm{CB})$ bottom at 
$\Gamma$-point. Interestingly, the valence band presents an inverted Mexican-hat-like dispersion, with band extremum located along the $\Gamma-\mathrm{M}$ and $\Gamma-\mathrm{K}$ directions.

In general, this peculiar valence band dispersion results in a divergence in the density of states, corresponding to the so-called van Hove singularities. When the Fermi level lies close to these singularities, the very large electronic density of states favors the occurrence of a ferromagnetic order $[44,45,46]$. Consequently, hole doping in InSe monolayer should result in a ferromagnetic material, like in GaS and GaSe monolayers [34,35].

To verify this concept, we considered a charged $5 \times 5 \times 1$ supercell and we calculated the magnetic moment and the spin polarization energy by increasing the hole density (n). Note that the spin polarization energy was defined as the energy difference between the non-magnetic and the ferromagnetic state, and positive values reveal the preference for the ferromagnetic state. As shown in Fig.2, a ferromagnetic state spontaneously emerges even at low hole densities and the magnetic moment rapidly reaches the saturation value of $1 \mu_{\mathrm{B}} /$ carrier. The magnetic moment per carrier remains constant up to $n \approx 1.5 \times 10^{14} / \mathrm{cm}^{2}$, whereas further increase of the hole density leads to the reduction of the magnetic moment. On the other hand, the spin polarization energy strongly depends on the doping level. Starting from low hole densities, it increases by increasing the density. For $n \approx 1 \times 10^{14} / \mathrm{cm}^{2}$, it reaches the maximum value of $11.5 \mathrm{meV} /$ carrier and for $n>1.0 \times 10^{14} / \mathrm{cm}^{2}$ it gradually decreases until it becomes zero. It is worth noting that for charged slabs, the electrostatic potential diverges with the distance from the slab, and the total energy depends on the thickness of the vacuum. Our test calculations showed that by increasing the vacuum thickness, although the energy significantly increases, the energy difference between the non-magnetic and the ferromagnetic states (which is used to define the polarization energy) 
remains almost unaffected.

Next, we computed the spin-polarized band structures of charged supercells at various hole densities. As shown in Fig.3, for a small amount of hole doping $\left(n=0.6 \times 10^{13} / \mathrm{cm}^{2}\right)$, an exchange splitting of the opposite-spin states near the Fermi level is observed. By increasing the hole density $\left(n=1.0 \times 10^{14} / \mathrm{cm}^{2}\right)$, the energy difference between the spin up and spin down valence band maxima significantly increases, whereas the Fermi level is slightly shifted downwards. This leads to a half metallic behavior where the spin up and spin down states present "insulating" and "metallic" nature, respectively. By increasing further the hole density $(n=$ $1.7 \times 10^{14} / \mathrm{cm}^{2}$ ), the Fermi level crosses both the spin up and spin down states, and the half metallic behavior is vanished.

Similar to InSe, both GaSe and GaS monolayers present an inverted Mexican-hat like valence band dispersion which leads to the Stoner-type ferromagnetism upon hole doping [34,35]. The ferromagnetic to the non-magnetic phase transition occurs at about $n=1.3-1.5 \times 10^{14} / \mathrm{cm}^{2}$ and $n=2.6 \times 10^{14} / \mathrm{cm}^{2}$ for GaSe and GaS, respectively, and similar to InSe, half-metallicity has been observed in hole-doped GaSe [35,47]. Overall, single-layer InSe appears to be as promising as GaSe and GaS for next generation spintronic nanodevices.

Next, we study the impact of hole doping in bilayer InSe. As shown in Fig.4, we consider various configurations with different stacking patterns namely 2L-I, 2L-II, 2L-III, 2L-IV, and 2L-V. Although their in plane lattice constants are similar, the interlayer distances of 2L-I, 2L-III and $2 \mathrm{~L}-\mathrm{V}$ configurations are lower compared to those of 2L-II and 2L-IV. In addition, 2L-I, 2L-III and $2 \mathrm{~L}-\mathrm{V}$ structures present total energies which are lower compared to those of $2 \mathrm{~L}-\mathrm{II}$ and $2 \mathrm{~L}-\mathrm{V}$ (see Supporting Information, Table SI). Regarding the electronic properties, Fig.5 shows the 
topmost valence band of various configurations and Table I summarizes their so-called Mexican hat parameters $\varepsilon 0$ and ko, which correspond to the Mexican-hat depth and width, respectively.

Table I: Mexican-hat parameters of bilayer InSe with various stacking patterns. For the sake of comparison, the corresponding values for monolayer InSe are also presented.

\begin{tabular}{ccc}
\hline Structure & $\varepsilon_{\mathrm{o}}(\mathrm{meV})$ & $\mathrm{k}_{\mathrm{o}}\left(\mathrm{nm}^{-1}\right)$ \\
\hline 1L & 71.9 & 2.7 \\
2L-I & 54.3 & 1.9 \\
2L-II & 51.5 & 2.1 \\
2L-III & 56.3 & 1.9 \\
2L-IV & 50.7 & 2.1 \\
2L-V & 55.6 & 1.9 \\
\hline
\end{tabular}

Going from monolayer to bilayer, the size of the Mexican-hat decreases. Taking into account that the sombrero-shape valence band is responsible for the Stoner-type ferromagnetism, its size will significantly affect the hole doping induced ferromagnetism. To verify this concept, for the case of $2 \mathrm{~L}-\mathrm{I}$, which is among the energetically favourable configurations, we calculated the magnetic moment by increasing the hole density (see Supporting Information, Figure S1). As expected, hole doping induced ferromagnetism is less pronounced in the bilayer InSe as compared to the monolayer.

As a next step, we computed the band structure of multilayer InSe. Figure 6 shows the topmost valence band of various configurations with an increasing thickness, and Table II summarizes their Mexican-hat parameters. 
Table II: Mexican-hat parameters of $\beta$-InSe by increasing its thickness.

\begin{tabular}{ccc}
\hline Structure & $\varepsilon_{\mathrm{o}}(\mathrm{meV})$ & $\mathrm{k}_{\mathrm{o}}\left(\mathrm{nm}^{-1}\right)$ \\
\hline 1L, 2L & $71.9,54.3$ & $2.7,1.9$ \\
3L, 4L & $43.4,31.8$ & $1.5,1.3$ \\
5L, 6L & $24.5,18.5$ & $1.3,1.0$ \\
7L, 8L & $13.6,10.5$ & $1.0,0.8$ \\
9L, 10L & $7.9,6.2$ & $0.8,0.8$ \\
12L, 14L & $3.3,1.9$ & $0.6,0.6$ \\
20L & 0.1 & 0.2 \\
\hline
\end{tabular}

As the thickness of InSe increases, the Mexican-hat size significantly decreases. For InSe consisting of 20 layers, similar to bulk InSe, the valence band is almost parabolic, and no ferromagnetism upon hole doping is expected. It is worth noting that in our study we considered the $\beta$-phase of InSe. The electronic structure of $\gamma$-InSe with an increasing thickness has been reported by Mudd et al. [48]. Similar to $\beta$-InSe, as the thickness of $\gamma$-InSe decreases the valence band top moves away from the $\Gamma$-point, and the valence band takes the shape of an inverted Mexican-hat [48].

Overall, our simulations reveal that although single-layer InSe is intrinsically non-magnetic, a stable ferromagnetic phase appears for a wide range of hole densities. Interestingly, hole doping induces not only spontaneous magnetization but also half-metallicity, and hole-doped InSe presenting one conducting and one insulating spin channel could be highly promising for next generation spintronic nanodevices.

3.2 Hole-doping induced by intrinsic defects 
As a next step, we examine the possibility to induce hole-doping and a subsequent ferromagnetic order by intrinsic defects. We consider a variety of structural imperfections in the neutral charge state, including mono-vacancies $\left(\mathrm{V}_{\mathrm{Se}}, \mathrm{V}_{\mathrm{In}}\right)$, di-vacancies $\left(\mathrm{V}_{\mathrm{SeIn}}, \mathrm{V}_{\mathrm{Se} 2}, \mathrm{~V}_{\mathrm{In} 2}\right)$, and antisites $\left(\mathrm{Se}_{\mathrm{In}}\right.$, $\mathrm{In}_{\mathrm{Se}}$ ), and their relaxed atomic structures are shown in Fig.7. As observed in this figure, single vacancies cause a moderate distortion of the lattice, whereas Se-In di-vacancy leads to a significant reconstruction. In particular, Se and In atoms neighboring the In vacant site relax inwards by $28.5 \%$ and $29.2 \%$, respectively, which results in the formation of two In-Se bonds of length $2.87 \AA$. Accordingly, In atoms neighboring the Se vacant site undergo an inward relaxation of $11.9 \%$, leading to the formation of one In-In bond of length $3.34 \AA$. For Se and In di-vacancies, under-coordinated atoms move towards the vacant sites by an average of $8.8 \%$ and $10.6 \%$, respectively. Note that for e.g. Se di-vacancy various configurations were studied, where the missing lattice sites were located either in the same or in different layers, and the distance between them varied. Among them, the energetically favorable (or most stable) was presented. Regarding the antisites, Se substituent forms three weak bonds with surrounding Se atoms of length $2.80 \AA$, i.e. longer compared to the bonds of bulk Se, and one In-Se bond of length 2.72 $\AA$. On the other hand, In substituent binds to three In atoms and the In-In bond length is $2.77 \AA$. To evaluate the thermodynamic stability of these defects, we calculate their formation energies in a wide range of Se chemical potential values. For the growth of bulk InSe we have $\mu_{\text {InSe }}^{S}=\mu_{\text {In }}+$ $\mu_{\text {Se }}$ where $\mu_{\text {In }}$ and $\mu_{\text {Se }}$ are the chemical potentials of In and Se atoms, respectively. The heat of formation is $\Delta \mathrm{H}_{\mathrm{InSe}}^{\mathrm{f}}=\mu_{\mathrm{InSe}}^{\mathrm{s}}-\mu_{\mathrm{In}}^{\mathrm{s}}-\mu_{\mathrm{Se}}^{\mathrm{s}}$ where $\mu_{\mathrm{InSe}}^{\mathrm{s}}$ is the chemical potential of solid InSe, whereas $\mu_{\mathrm{In}}^{\mathrm{S}}$ and $\mu_{\mathrm{Se}}^{\mathrm{S}}$ are the chemical potentials of solid In and Se in their ground state structures, respectively. The heat of formation is found to be $\Delta \mathrm{H}_{\mathrm{InSe}}^{\mathrm{f}}=-1.34 \mathrm{eV}$, and the 
negative value confirms the stability of InSe against pure In and Se structures. By taking into account that $\mu_{\mathrm{In}}<\mu_{\mathrm{In}}^{\mathrm{S}}$ and $\mu_{\mathrm{Se}}<\mu_{\mathrm{Se}}^{\mathrm{s}}$ we obtain the upper and lower limits for each potential $\mu_{\text {In }}^{\mathrm{s}}+\Delta \mathrm{H}_{\text {InSe }}^{\mathrm{f}}<\mu_{\mathrm{In}}<\mu_{\mathrm{In}}^{\mathrm{s}}$ and $\mu_{\mathrm{Se}}^{\mathrm{s}}+\Delta \mathrm{H}_{\mathrm{InSe}}^{\mathrm{f}}<\mu_{\mathrm{Se}}<\mu_{\mathrm{Se}}^{\mathrm{s}}$. By considering a parameter $\lambda(0 \leq$ $\lambda \leq 1)$ we have $\mu_{\text {In }}=\mu_{\text {In }}^{\mathrm{s}}+(1-\lambda) \Delta \mathrm{H}_{\mathrm{InSe}}^{\mathrm{f}}$ and $\mu_{\mathrm{Se}}=\mu_{\mathrm{Se}}^{\mathrm{s}}+\lambda \Delta \mathrm{H}_{\mathrm{InSe}}^{\mathrm{f}}$, where $\lambda=0$ and $\lambda=1$ correspond to Se-rich and In-rich conditions, respectively.

If $\Delta \mu=\left(\mu_{\text {In }}-\mu_{\text {In }}^{\mathrm{S}}\right)-\left(\mu_{\mathrm{Se}}-\mu_{\mathrm{Se}}^{\mathrm{S}}\right)=\left(\mu_{\mathrm{In}}-\mu_{\mathrm{Se}}\right)-\left(\mu_{\mathrm{In}}^{\mathrm{S}}-\mu_{\mathrm{Se}}^{\mathrm{S}}\right)$ and consequently $\Delta \mu=$ $(1-2 \lambda) \Delta \mathrm{H}_{\mathrm{InSe}}^{\mathrm{f}}, \Delta \mu$ is restricted in the range $-\Delta \mathrm{H}_{\mathrm{InSe}}^{\mathrm{f}} \leq \Delta \mu \leq \Delta \mathrm{H}_{\text {InSe }}^{\mathrm{f}}$. Finally, the formation energy of a defect at zero temperature in a neutral charge state is given by the equation [49]

$E_{\text {for }}\left(\mu_{\text {In }}, \mu_{\mathrm{Se}}\right)=E_{\text {def }}-\frac{1}{2}\left(n_{\text {In }}+n_{\text {Se }}\right) \mu_{\text {InSe }}^{\mathrm{s}}-\frac{1}{2}\left(n_{\text {In }}-n_{\text {Se }}\right)\left(\mu_{\text {In }}^{\mathrm{s}}-\mu_{\text {Se }}^{\mathrm{s}}\right)-\frac{1}{2}\left(n_{\text {In }}-n_{\text {Se }}\right) \Delta \mu$

where $n_{\text {In }}$ and $n_{\text {Se }}$ are the number of In and Se atoms in the defective structure, respectively.

Figure 8 shows the thermodynamic stability diagram of defects' formation energies, taking as reference energy the ground state energy of the pristine system. All defect formation energies are found to be positive, similar to other widely studied two-dimensional materials $[50,51,52,53,54,55]$. The positive values indicate the energy cost for creating the defect, and the larger the defect formation energy is, the more difficult to create the defect.

As observed in this figure, under In-rich conditions the lowest formation energy corresponds to Se vacancy which is $2.33 \mathrm{eV}$ lower than that of metal vacancy. Regarding the di-vacancies, $\mathrm{V}_{\mathrm{Se} 2}$ formation energy is almost double that of $\mathrm{V}_{\mathrm{Se}}$ whereas an energy gain of $\mathrm{E}_{\text {for }}\left(\mathrm{V}_{\text {SeIn }}\right)-$ $\left[E_{\text {for }}\left(V_{\text {In }}\right)+E_{\text {for }}\left(V_{S e}\right)\right]=-1.74 \mathrm{eV}$ is found for $V_{\text {SeIn. }}$ On the other hand, under Se-rich conditions, Se antisite and In vacancy are found to be favorable with formation energies 1.33 and $2.03 \mathrm{eV}$, respectively. It is worth noting that in a wide range of Se chemical potential values, 
from moderate Se-rich stoichiometric growth and up to extreme In-rich conditions, the lowest formation energy corresponds to Se vacancy, whereas the formation of In antisite and Se divacancy requires roughly $80-90$ and $100 \%$ additional energy, respectively.

Next, the impact of defects on the electronic properties of InSe monolayers is examined. The computed band structures of defective systems and the partial charge densities of defect-related states are shown in Fig.9. As observed in this figure, Se vacancy creates one occupied and two empty states within the gap. Interestingly, one almost degenerate state close to the $\mathrm{CB}$ and spin polarized states close to the VB are found for In vacancy. The spin-polarized states shift the Fermi level near the VB and lead to a p-type behavior. Our projected DOS calculations show that spin-down defect states close to the valence band are mainly derived from the $4 p$ orbitals of under-coordianted Se atoms, neighboring the vacant site (see Supporting Information, Figure S2). Taking into account that $\mathrm{V}_{\text {In }}$ formation energy is relatively low in the Se-rich limit, InSe monolayers grown under Se-rich conditions may be ferromagnetic and potentially useful for spin-based field effect transistors.

Regarding the di-vacancies, $\mathrm{V}_{\text {SeIn }}$ and $\mathrm{V}_{\text {Se2 }}$ create several gap states, whereas $\mathrm{V}_{\operatorname{In} 2}$ acts as acceptor and results in a p-type behavior. Note that for $\mathrm{V}_{\text {In2 }}$ defect the FM and AFM configurations present very similar energies, and the band structure of the FM configuration is presented. For both antisites, spin-polarized gap states and a total magnetic moment of $1 \mu_{\mathrm{B}}$ are observed.

For single vacancies, we additionally performed hybrid functional calculations using the HSE functional (see Supporting Information, Figure S3 ). Pristine InSe monolayer is found to be a semiconductor with a computed indirect gap of $2.28 \mathrm{eV}$, in good agreement with previously reported HSE calculations [42,56,57]. Similar to PBE calculations, HSE calculations revealed 
that Se vacancy creates three gap states which lie $\sim 0.1$ and $2.2 \mathrm{eV}$ below the CB bottom. For In vacancy, one degenerate level $\sim 0.4 \mathrm{eV}$ below the $\mathrm{CB}$ and two spin polarized levels $\sim 1.9 \mathrm{eV}$ below the CB are introduced. Thus, despite of the gap opening, the defect states remain close to the VB and the Fermi level remains pinned close to the VB.

Lastly, for the case of In vacancy where hole-doping induced ferromagnetism is observed, we performed first principles molecular dynamics simulations at $\mathrm{T}=300 \mathrm{~K}$, in order to check the stability of the defective system and if ferromagnetism survives at room temperature. After 5 ps, the saturation of two (out of three) Se dangling bonds is observed, whereas the ground state remains ferromagnetic with an average magnetic moment of $0.9 \mu_{\mathrm{B}}$ (see Supporting Information, S4). For the defective configuration obtained after MD simulations, we additionally computed its band structure. Similar to our previous analysis, we found defect states close to the VB which shift and pin the Fermi level close to the VB and lead to a p-type behaviour.

\subsection{Hole-doping induced by extrinsic defects}

Next, we consider a $5 \times 5 \times 1$ supercell and we study the substitution of a Se atom by a group-V atom, namely $\mathrm{P}, \mathrm{As}, \mathrm{Sb}$, and $\mathrm{Bi}$. Since Se atoms contribute six valence electrons whereas group$\mathrm{V}$ atoms contribute only five electrons, such substitutional impurities are good candidates to induce hole doping and a subsequent ferromagnetic order. The relaxed atomic structures and the computed band structures are shown in Fig.10a-d. As observed in this figure, In atoms neighboring the $\mathrm{P}$ and As dopants relax inwards by 4.9 and $2.0 \%$, resulting in shortened P-In and As-In bonds of 2.55 and $2.62 \AA$, respectively. On the contrary, In atoms surrounding the Sb and Bi dopants display an outward relaxation of $\sim 4.1$ and $6.6 \%$, leading to elongated Sb-In and 
Bi-In bonds of 2.79 and $2.85 \AA$, respectively.

To evaluate the thermodynamic stability of these dopants, we calculate their formation energies under different growth conditions using the equation

$\mathrm{E}_{\text {for }}=\mathrm{E}_{\text {tot }}(\mathrm{InSe}+\mathrm{X})-\mathrm{E}_{\mathrm{tot}}(\mathrm{InSe})+\mu_{\mathrm{H}}-\mu_{\mathrm{X}}$

where $E_{\text {tot }}(\operatorname{InSe}+X), E_{\text {tot }}(\operatorname{InSe})$ are the total energies of doped and pristine systems, and $\mu_{H}$, $\mu_{\mathrm{X}}$ are the chemical potentials of host and doped atoms, respectively. Note that $\mu_{\mathrm{H}}$ strongly depends on the growth conditions, and under In-rich conditions we have $\mu_{\text {In }}^{\text {In-rich }}=\mu_{\text {In }}^{S}$, $\mu_{\mathrm{Se}}^{\mathrm{In}-\mathrm{rich}}=\Delta \mathrm{H}_{\mathrm{f}}+\mu_{\mathrm{Se}}^{\mathrm{S}}$, whereas under Se-rich conditions we have $\mu_{\mathrm{In}}^{\mathrm{Se}-\mathrm{rich}}=\Delta \mathrm{H}_{\mathrm{f}}+\mu_{\mathrm{In}}^{\mathrm{S}}$, $\mu_{\mathrm{Se}}^{\mathrm{Se}-\text { rich }}=\mu_{\mathrm{Se}}^{\mathrm{S}}$. As shown in Table III, the computed formation energies are relatively low under In-rich conditions. In particular, the lowest formation energy corresponds to As, which is about $0.18,0.24$, and $0.36 \mathrm{eV}$ lower compared to that of $\mathrm{P}, \mathrm{Sb}$, and $\mathrm{Bi}$, respectively.

As a representative system, the magnetic and electronic properties of Bi-doped InSe are mainly discussed. Bi doping results in a magnetic ground state with a total magnetic moment of $1 \mu_{\mathrm{B}}$. The energy difference between the non-magnetic and the ferromagnetic phase is $0.05 \mathrm{eV}$, where the positive value reveals the preference for the ferromagnetic phase. Regarding the electronic properties, Bi creates spin polarized states within the gap. More precisely, we observe one occupied and one empty state, located 1.35 and $1.15 \mathrm{eV}$ below the conduction band bottom. A similar behavior is also observed for $\mathrm{P}, \mathrm{As}$, and $\mathrm{Sb}$ dopants. By increasing the dopant atomic number, the unoccupied states move toward the valence band. Also, the spin splitting of the gap states reduces, going from $\sim 0.4 \mathrm{eV}$ for P-doping to $\sim 0.2 \mathrm{eV}$ for $\mathrm{Bi}$ doping. Interestingly, the dependence of the spin splitting on the atomic number follows the trend for the exchange correlation integral $[58,59]$. Overall, among all considered impurities, $\mathrm{Bi}$ is the most promising 
one to induce p-type conductivity, since the unoccupied impurity state lies very close to the valence band top. Note that our projected DOS calculations show that for all group-V doped systems, the opposite spin states close to the valence band are mainly derived from the group-V $\mathrm{p}$ orbitals and $5 \mathrm{p}$ orbitals of In atoms neighboring the impurity atom (see Supporting Information, Figure S5).

Next, for all systems, we examine if ferromagnetism survives at room temperature using firstprinciples molecular dynamics simulations at $\mathrm{T}=300 \mathrm{~K}$. After $5 \mathrm{ps,}$ only minor fluctuations in the magnetic moments are observed, and the ground states remain ferromagnetic with an average magnetic moment of $1.0 \mu_{\mathrm{B}}$ (see Supporting Information, Figure S6).

Lastly, for the sake of completeness, the substitution of an In atom by a group-V atom is examined. As shown in Table III, the formation energies of In substituents are significantly larger compared to those of Se-substituents in the In-rich (or Se-poor) limit. Regarding the electronic properties, although gap states are observed, the Fermi level is no longer located close to the valence band and the systems are found to be non-magnetic, as shown in Fig.10e-h.

Table III: Computed formation energies $\left(\mathrm{E}_{\text {for }}\right)$, total magnetic moments $\left(\mathrm{M}_{\mathrm{tot}}\right)$, and spin polarization energies $\left(\mathrm{E}_{\mathrm{pol}}\right)$ of various substitutional impurities.

\begin{tabular}{cccccc}
\hline System & $\mathrm{E}_{\text {for }}$ In-rich $(\mathrm{eV})$ & $\mathrm{E}_{\text {for }}$ Se-rich $(\mathrm{eV})$ & Ground State & $\mathrm{M}_{\text {tot }}\left(\mu_{\mathrm{B}}\right)$ & $\mathrm{E}_{\mathrm{pol}}(\mathrm{eV})$ \\
\hline $\mathrm{In}_{50} \mathrm{Se}_{49} \mathrm{P}$ & 0.60 & 1.94 & FM & 1.0 & 0.09 \\
\hline
\end{tabular}




\begin{tabular}{cccccc}
\hline $\mathrm{In}_{50} \mathrm{Se}_{49} \mathrm{As}$ & 0.42 & 1.76 & FM & 1.0 & 0.07 \\
$\mathrm{In}_{50} \mathrm{Se}_{49} \mathrm{Sb}$ & 0.66 & 2.00 & $\mathrm{FM}$ & 1.0 & 0.07 \\
$\mathrm{In}_{50} \mathrm{Se}_{49} \mathrm{Bi}$ & 0.78 & 2.12 & $\mathrm{FM}$ & 1.0 & 0.05 \\
$\mathrm{In}_{49} \mathrm{Se}_{50} \mathrm{P}$ & 2.36 & 1.02 & $\mathrm{NM}$ & 0.0 & 0.00 \\
$\mathrm{In}_{49} \mathrm{Se}_{50} \mathrm{As}$ & 1.76 & 0.42 & $\mathrm{NM}$ & 0.0 & 0.00 \\
$\mathrm{In}_{49} \mathrm{Se}_{50} \mathrm{Sb}$ & 1.67 & 0.34 & $\mathrm{NM}$ & 0.0 & 0.00 \\
$\mathrm{In}_{49} \mathrm{Se}_{50} \mathrm{Bi}$ & 1.24 & -0.10 & $\mathrm{NM}$ & 0.0 & 0.00 \\
\hline
\end{tabular}

\section{Conclusions}

In this paper, we performed first-principles calculations based on density functional theory to study the magnetic and electronic properties of hole-doped two-dimensional InSe. Our simulations revealed that although $2 \mathrm{D}$ InSe is intrinsically non-magnetic, a stable ferromagnetic phase appears for a wide range of hole densities. Interestingly, hole doping not only induces spontaneous magnetization but also half-metallicity, and hole-doped InSe, presenting one conducting and one insulating spin channel, could be highly promising for next generation spintronic nanodevices. The possibility to induce hole doping by intrinsic defects was also investigated. We found that In vacancy creates spin polarized states close to the valence band and leads to a p-type behavior. Taking into account that $\mathrm{V}_{\text {In }}$ formation energy is relatively low in the Se-rich limit, InSe monolayers grown under Se-rich conditions may be ferromagnetic and potentially useful for spin-based nanoelectronics. As a last step, hole doping resulting from substitutional doping was investigated. Similar to In vacancies, group-V atoms replacing Se atoms create spin polarized states close to the valence band. By increasing the dopant atomic number, their spin splitting reduces and the unoccupied states move toward the valence band, potentially stabilizing the ferromagnetic order in $2 \mathrm{D}$ InSe. In this case, a proposed doping strategy consists in growing samples under In-rich conditions to produce Se vacancies (with a 
relatively low formation energy), and subsequently to dope the material with group- $\mathrm{V}$ atoms, that would fill the Se vacant sites and become electrically active. On the other hand, group-V atoms replacing In atoms induce deeper states within the gap, and no magnetism is observed.

\section{Acknowledgements}

This work has been financially supported by the KU Leuven Research Funds, project GOA/13/011, the 2Dfun project (2D functional MX2/graphene hetero-structures), an ERA-NET project in the framework of the Graphene Flagship, and the Research Council of Norway (ToppForsk project: 251131). The authors acknowledge the support from Flanders Innovation \& Entrepreneurship. Part of this work was supported by computational time granted from the Greek Research \& Technology Network (GRNET) in the National HPC facility ARIS under the project AMONADE (pr004002). Also, we acknowledge access to HPC resources Abel and Fram in Norway with allocation provided through NOTUR.

\section{References}

[1] M. Ieong, B. Doris, J. Kedzierski, and Y. Min, "Silicon Device Scaling to the Sub-10-nm Regime." Science, 306, 2057-2060 (2004).

[2] S. Z. Butler, S. M. Hollen, L. Cao, Y. Cui, J. A. Gupta, H. R. Gutiérrez, T. F. Heinz, S. S. Hong, J. Huang, A. F. Ismach, and E. Johnston-Halperin, "Progress, Challenges and Opportunities in Two-Dimensional Materials Beyond Graphene.” ACS Nano, 7, 2898-2926 (2013).

[3] G. Fiori, F. Bonaccorso, G. Iannaccone, T. Palacios, D. Neumaier, A. Seabaugh, S. K. Banerjee, and L. Colombo, "Electronics Based on Two-Dimensional Materials.", Nat. Nanotechnol., 9, 768-779 (2014). 
[4] F. Schwierz, J. Pezoldt, and R. Granzner, "Two-Dimensional Materials and their Prospects in Transistor Electronics.” Nanoscale, 7, 8261-8283 (2015).

[5] M. Houssa, A. Dimoulas, and A. Molle, "Silicene: A Review of Recent Experimental and Theoretical Investigations.” J. Phys.: Condens. Matter, 27, 253002 (2015).

[6] E. Scalise, K. Iordanidou, V. V. Afanas'ev, A. Stesmans, and M. Houssa, "Silicene on NonMetallic Substrates: Recent Theoretical and Experimental Advances.” Nano Res., 1-14 (2017).

[7] B. van den Broek, M. Houssa, K. Iordanidou, G. Pourtois, V. V. Afanas'ev, and A. Stesmans, "Functional Silicene and Stanene Nanoribbons Compared to Graphene: Electronic Structure and Transport." 2D Mater., 3, 015001 (2016).

[8] K. S. Novoselov, A. K. Geim, S. V. Morozov, D. Jiang, Y. Zhang, S. V. Dubonos, I. V. Grigorieva, and A. A. Firsov, "Electric Field Effect in Atomically Thin Carbon Films." Science, 306, 666-669 (2004).

[9] A. Politano, G. Chiarello, R. Samnakay, G. Liu, B. Gürbulak, S. Duman, A. A. Balandin, and D. W. Boukhvalov, "The Influence of Chemical Reactivity of Surface Defects on Ambient-Stable InSe-Based Nanodevices.” Nanoscale, 8, 8474-8479 (2016).

[10] X. Li, C. Xia, X. Song, J. Du, and W. Xiong, "n-and p-Type Dopants in the InSe Monolayer via Substitutional Doping.” J. Mater. Sci., 52, 7207-7214 (2017).

[11] T. Hu, J. Zhou, and J. Dong, "Strain Induced New Phase and Indirect-Direct Band Gap Transition of Monolayer InSe.” Phys. Chem. Chem. Phys., 19, 21722-21728 (2017).

[12] X. Liu, J. C. Ren, S. Zhang, M. Fuentes-Cabrera, S. Li, and W. Liu, "Ultrahigh Conductivity in Two-Dimensional InSe via Remote Doping at Room Temperature.” J. Phys. Chem. Lett., 9, 3897-3903 (2018).

[13] P. H. Ho, Y. R. Chang, Y. C. Chu, M. K. Li, C. A. Tsai, W. H. Wang, ... and P. W. Chiu, "High-Mobility InSe Transistors: The Role of Surface Oxides" ACS Nano, 11, 7362-7370 (2017).

[14] Z. Fu, B. Yang, N. Zhang, Z. Lu, Z. Yang, and D. Ma, "Tuning the Physical and Chemical Properties of 2D InSe with Interstitial Boron Doping: A First-Principles Study.” J. Phys. Chem. C, 121, 28312-28316(2017). 
[15] Y. Wang, R. Fei, R. Quhe, J. Li, H. Zhang, X. Zhang,... and J. J. Shi, "Many-Body Effect and Device Performance Limit of Monolayer InSe" ACS Appl. Mater. Interfaces 10, 2334423352 (2018).

[16] X. Wei, C. Dong, A. Xu, X. Li, and D. D. Macdonald, "Oxygen-Induced Degradation of the Electronic Properties of Thin-Layer InSe." Phys. Chem. Chem. Phys., 20, 2238-2250 (2018).

[17] E. G. Marin, D. Marian, G. Iannaccone, and G. Fiori, "First-Principles Simulations of FETs Based on Two-Dimensional InSe.” IEEE Electron Device Lett., 39, 626-629 (2018).

[18] J. Zhou, J. Shi, Q. Zeng, Y. Chen, L. Niu, F. Liu, T. Yu, K. Suenaga, X. Liu, J. Lin, and Z. Liu, "InSe Monolayer: Synthesis, Structure and Ultra-High Second-Harmonic Generation." 2D Mater., 5, 025019 (2018).

[19] G. W. Mudd, S. A. Svatek, T. Ren, A. Patanè, O. Makarovsky, L. Eaves, P. H. Beton, Z. D. Kovalyuk, G. V. Lashkarev, Z. R. Kudrynskyi, and A. I. Dmitriev, "Tuning the Bandgap of Exfoliated InSe Nanosheets by Quantum Confinement.” Adv. Mater., 25, 5714-5718 (2013).

[20] D. A. Bandurin, A. V. Tyurnina, G. L. Yu, A. Mishchenko, V. Zólyomi, S. V. Morozov, R. K. Kumar, R. V. Gorbachev, Z. R. Kudrynskyi, S. Pezzini, et al. "High Electron Mobility, Quantum Hall Effect and Anomalous Optical Response in Atomically Thin InSe" Nat. Nanotechnol., 12, 223 (2016).

[21] J. Lauth, F. E. Gorris, M. Samadi Khoshkhoo, T. Chassé, W. Friedrich, V. Lebedeva, A. Meyer, C. Klinke, A. Kornowski, M. Scheele, and H. Weller, "Solution-Processed TwoDimensional Ultrathin InSe Nanosheets." Chem. Mater., 28, 1728-1736 (2016).

[22] W. Feng, W. Zheng, W. Cao, and P. Hu, "Back Gated Multilayer InSe Transistors with Enhanced Carrier Mobilities via the Suppression of Carrier Scattering from a Dielectric Interface" Adv. Mater., 26, 6587-6593 (2014).

[23] G. W. Mudd, A. Patanè, Z. R. Kudrynskyi, M. W. Fay, O. Makarovsky, L. Eaves, Z. D. Kovalyuk, V. Zólyomi, and V. Falko, "Quantum Confined Acceptors and Donors in InSe Nanosheets.” Appl. Phys. Lett., 105, 221909 (2014).

[24] J. E. Padilha, R. H. Miwa, A. J. da Silva, and A. Fazzio, “Two-Dimensional van der Waals pn Junction of InSe/Phosphorene.” Phys. Rev. B, 95, 195143 (2017). 
[25] C. X. Xia, J. Du, X. W. Huang, W. B. Xiao, W. Q. Xiong, T. X. Wang, Z. M. Wei, Y. Jia, J. J. Shi, and J. B. Li, “Two-Dimensional n-InSe/p-GeSe (SnS) van der Waals Heterojunctions: High Carrier Mobility and Broadband Performance.” Phys. Rev. B, 97, 115416 (2018).

[26] B. Chakraborty, and L. M. Ramaniah, "Exploring d $\mathrm{d}^{0}$ Magnetism in Doped $\mathrm{SnO}_{2}-\mathrm{A}$ First Principles DFT Study.” J. Magn. Magn. Mater., 385, 207-216 (2015).

[27] B. Chakraborty, and L. M. Ramaniah, 2014, April. "First Principles DFT Study of Ferromagnetism in $\mathrm{SnO}_{2}$ Induced by Doped Group 1A and 2A Non-Magnetic Elements $\mathrm{X}(\mathrm{X}=$ Li, Na, K, Be, Mg, Ca).” AIP Conf. Proc., 1591, 1604-1605 (2015).

[28] B. Chakraborty, and L. M. Ramaniah, "Room Temperature $\mathrm{d}^{0}$ Ferromagnetism in Hole Doped $\mathrm{Y}_{2} \mathrm{O}_{3}$ : Widening the Choice of Host to Tailor DMS.” J. Phys.: Condens. Matter, 28, 336001 (2016).

[29] B. Chakraborty, P. K. Nandi, Y. Kawazoe, and L. M. Ramaniah, "Room-Temperature d" Ferromagnetism in Carbon-Doped $\mathrm{Y}_{2} \mathrm{O}_{3}$ for Spintronic Applications: A Density Functional Theory Study.” Phys. Rev. B, 97, 184411 (2018).

[30] K. Yang, Y. Dai, B. Huang, and M. H. Whangbo, "On the Possibility of Ferromagnetism in Carbon-Doped Anatase $\mathrm{TiO}_{2} . ”$ Appl. Phys. Lett., 93, 132507 (2008).

[31] K. Yang, Y. Dai, B. Huang, and M. H. Whangbo, "Density Functional Studies of the Magnetic Properties in Nitrogen Doped TiO2.” Chem. Phys. Lett., 481, 99-102 (2009).

[32] K. Yang, Y. Dai, and B. Huang, "Density Functional Study of Boron-Doped Anatase TiO $2 . "$ J. Phys. Chem. C, 114, 19830-19834 (2010).

[33] K. Yang, R. Wu, L. Shen, Y. P. Feng, Y. Dai, and B. Huang, "Origin of $d^{0}$ Magnetism in IIVI and III-V Semiconductors by Substitutional Doping at Anion Site.” Phys. Rev. B, 81, 125211 (2010).

[34] S. Wu, X. Dai, H. Yu, H. Fan, J. Hu, and W. Yao, "Magnetisms in p-Type Monolayer Gallium Chalcogenides (GaSe, GaS).” arXiv:1409.4733.

[35] T. Cao, Z. Li, and S. G. Louie, "Tunable Magnetism and Half-Metallicity in Hole-Doped Monolayer GaSe.” Phys. Rev. Lett., 114, 236602 (2015).

[36] G. Kresse, and J. Furthmüller, "Efficient Iterative Schemes for Ab Initio Total-Energy 
Calculations Using a Plane-Wave Basis Set.” Phys. Rev. B, 54, 11169 (1996).

[37] G. Kresse, and J. Furthmüller, "Efficiency of Ab-Initio Total Energy Calculations for Metals and Semiconductors Using a Plane-Wave Basis Set.” Comput. Mater. Sci., 6, 15-50 (1996).

[38] P. E. Blöchl, "Projector Augmented-Wave Method” Phys. Rev. B, 50, 17953 (1994).

[39] J. P. Perdew, K. Burke, and M. Ernzerhof, "Generalized Gradient Approximation Made Simple." Phys. Rev. Lett., 77, 3865 (1996).

[40] S. Grimme, J. Antony, S. Ehrlich, and S. Krieg, "A Consistent and Accurate Ab Initio Parametrization of Density Functional Dispersion Correction (dft-d) for the 94 Elements H-Pu.", J. Chem. Phys., 132, 154104 (2010).

[41] J. Heyd, G. E. Scuseria, and M. Ernzerhof, "Hybrid Functionals Based on a Screened Coulomb Potential.” J. Chem. Phys., 118, 8207-8215 (2003).

[42] H. L. Zhuang, and R. G. Hennig, "Single-Layer Group-III Monochalcogenide Photocatalysts for Water Splitting." Chem. Mater., 25, 3232-3238 (2013).

[43] L. Debbichi, O. Eriksson, and S. Lebègue, "Two-Dimensional Indium Selenides Compounds: An Ab Initio Study.” J. Phys. Chem. Lett., 6, 3098-3103 (2015).

[44] L. Seixas, A. S. Rodin, A. Carvalho, and A. C. Neto, "Multiferroic Two-Dimensional Materials.” Phys. Rev. Lett., 116, 206803 (2016).

[45] M. Houssa, K. Iordanidou, G. Pourtois, V. V. Afanas' ev, and A. Stesmans, "Hole-Doping Induced Ferromagnetism in Monolayer SnO: A First-Principles Study.” ECS Trans., 80, 339-345 (2017).

[46] M. Houssa, K. Iordanidou, G. Pourtois, V. V. Afanas'ev, and A. Stesmans "Ferromagnetism in Two-Dimensional Hole-Doped SnO.” AIP Adv., 8, 055010 (2018).

[47] F. Li, X. Zhou, W. Feng, B. Fu, and Y. Yao, "Thickness-Dependent Magneto-Optical Effects in Hole-Doped GaS and GaSe Multilayers: A First-Principles Study.” New J. Phys., 20, 043048 (2018).

[48] G. W. Mudd, M. R. Molas, X. Chen, V. Zólyomi, K. Nogajewski, Z. R. Kudrynskyi, Z. D. Kovalyuk, G. Yusa, O. Makarovsky, L. Eaves, and M. Potemski, “The Direct-to-Indirect Band Gap Crossover in Two-Dimensional van der Waals Indium Selenide Crystals.” Sci. Rep. 6, 
39619 (2016).

[49] G. X. Qian, R. Martin, and D. Chadi "First-Principles Study of the Atomic Reconstructions and Energies of Ga-and As-Stabilized GaAs (100) Surfaces." Phys. Rev. B, 38, 7649 (1998).

[50] S. Haldar, H. Vovusha, M. K. Yadav, O. Eriksson, and B. Sanyal, "Systematic Study of Structural, Electronic, and Optical Properties of Atomic-Scale Defects in the Two-Dimensional Transition Metal Dichalcogenides $\mathrm{MX}_{2}$ (M= Mo, W; X= S, Se, Te)." Phys. Rev. B, 92, 235408 (2015).

[51] H. P. Komsa, and A. V. Krasheninnikov, "Native Defects in Bulk and Monolayer MoS 2 from First Principles.” Phys. Rev. B, 91, 125304 (2015).

[52] K. Iordanidou, M. Houssa, G. Pourtois, V. V. Afanas' ev, and A. Stesmans, "Impact of Point Defects and Oxidation on the Electronic Properties of $\mathrm{HfS}_{2}$ Monolayers." ECS J. Solid State Sci. Technol., 5, 3054-3059 (2016).

[53] F. Banhart, J. Kotakoski, and A. V. Krasheninnikov, "Structural Defects in Graphene." ACS Nano, 5, 26-41 (2010).

[54] K. Iordanidou, M. Houssa, B. van den Broek, G. Pourtois, V. V. Afanas'ev, and A. Stesmans, "Impact of Point Defects on the Electronic and Transport Properties of Silicene Nanoribbons." J. Phys.: Condens. Matter, 28, 035302 (2016).

[55] K. Iordanidou, J. Kioseoglou, V. V. Afanas'ev, A. Stesmans, and M. Houssa, "Intrinsic Point Defects in Buckled and Puckered Arsenene: A First-Principles Study.” Phys. Chem. Chem. Phys., 19, 9862-71 (2017).

[56] Q. Peng, R. Xiong, B. Sa, J. Zhou, C. Wen, B. Wu, M. Anpo, and Z. Sun, “Computational Mining of Photocatalysts for Water Splitting Hydrogen Production: Two-Dimensional InSeFamily Monolayers." Catal. Sci. Technol., 7, 2744-2752 (2017).

[57] Y. Guo, and J. Robertson, "Band Structure, Band Offsets, Substitutional Doping, and Schottky Barriers of Bulk and Monolayer InSe." Phys. Rev. Mater., 1, 044004 (2017).

[58] J. F. Janak, “Uniform Susceptibilities of Metallic Elements," Phys. Rev. B, 16, 255 (1977).

[59] K. Dolui, I. Rungger, C. D. Pemmaraju, and S. Sanvito, "Possible Doping Strategies for MoS 2 Monolayers: An Ab Initio Study.” Phys. Rev. B, 88, 075420 (2013). 
(a)

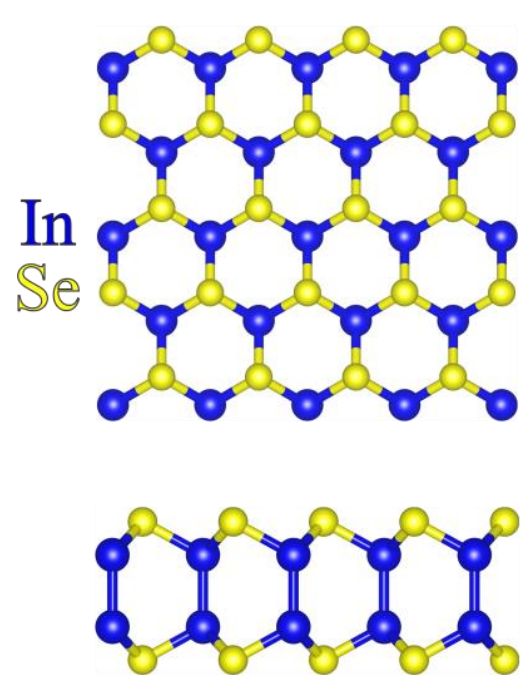

(b)

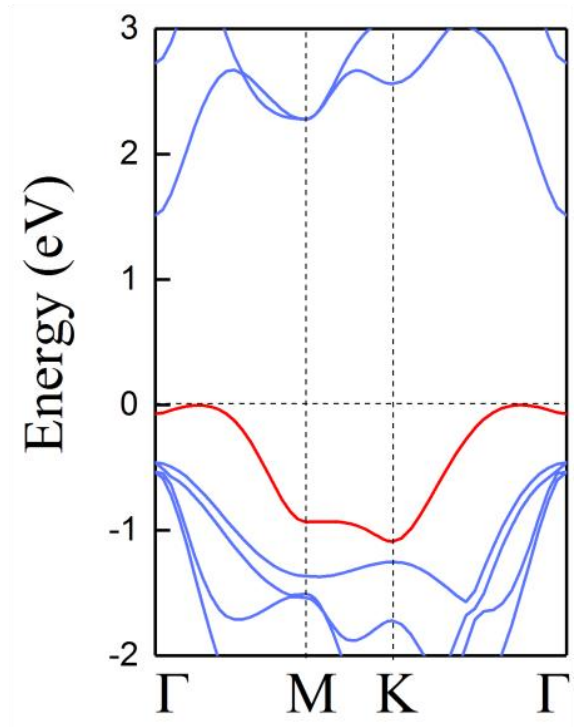

Figure 1: (a) Top and side views of the relaxed atomic structure of InSe monolayer. Blue and yellow spheres correspond to In and Se atoms, respectively. (b) Computed band structure of single-layer unit cell. The energies refer to the VB top. The VB presenting a Mexican-hat-like dispersion is highlighted in red. 


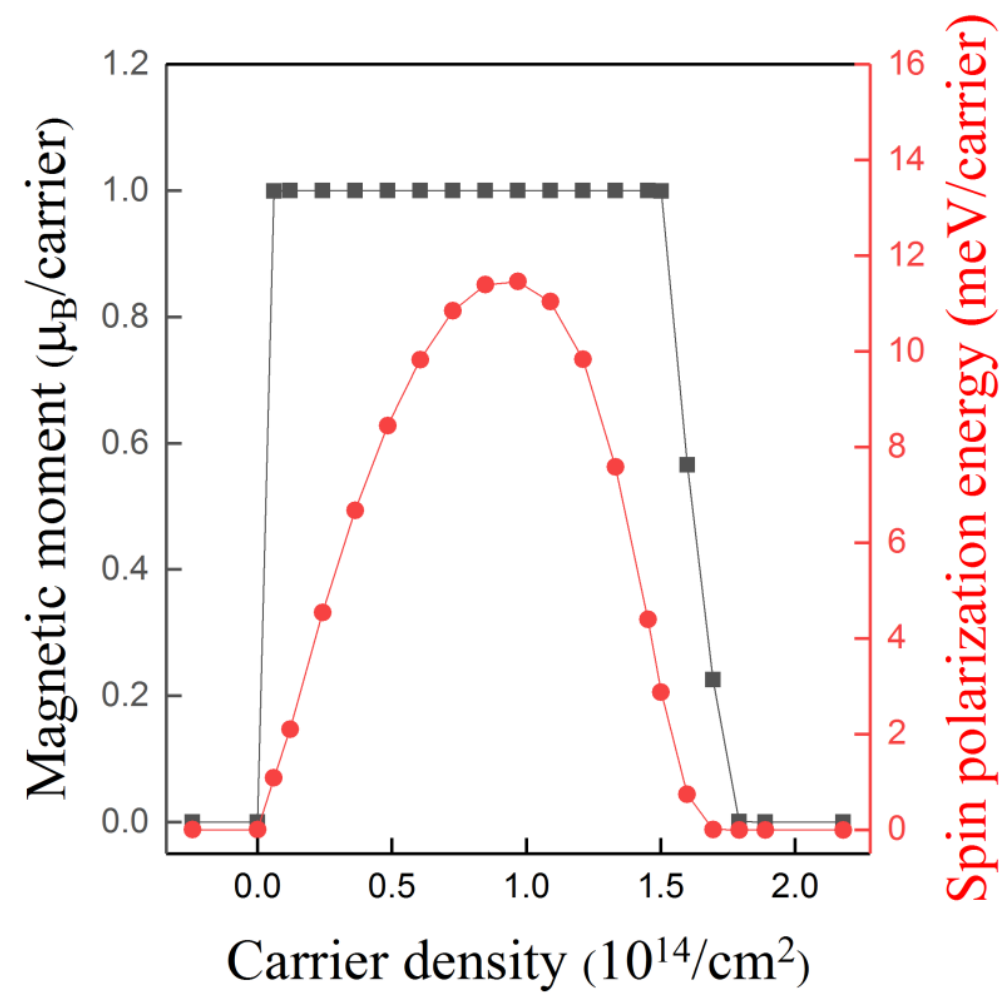

Figure 2: Magnetic moment and spin polarization energy as a function of the carrier density. The negative carrier density refers to the electron-doping case. 
(a)

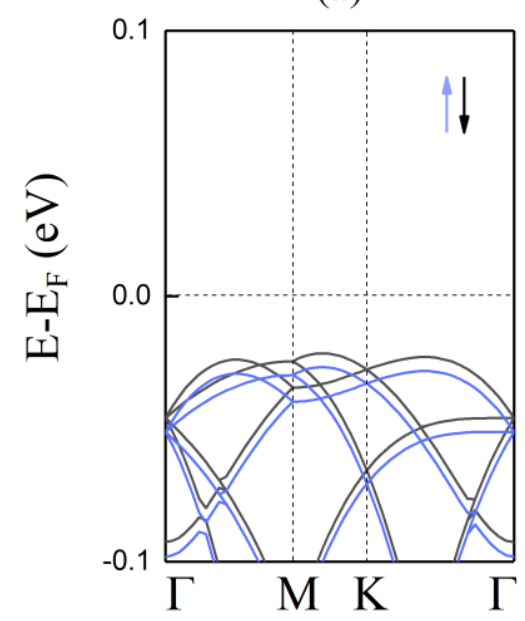

(b)

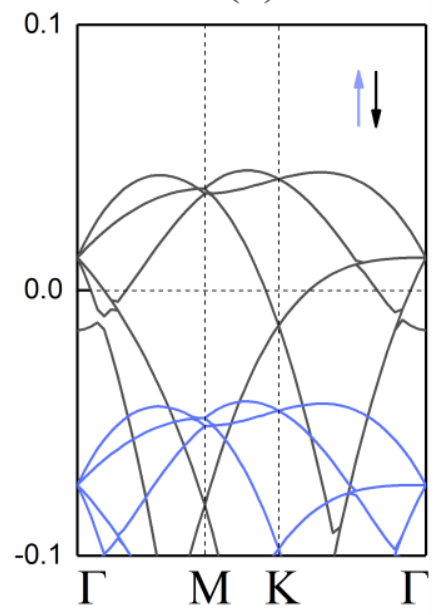

(c)

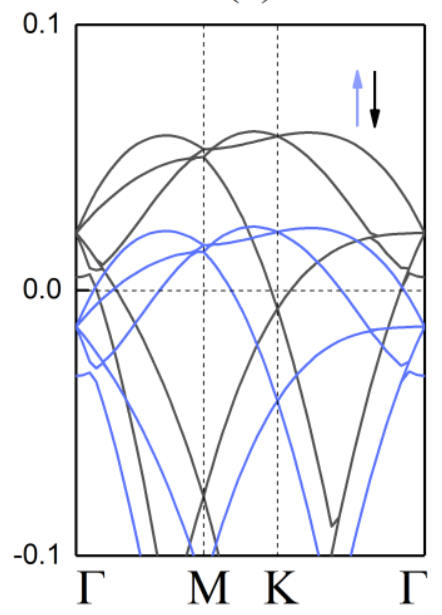

Figure 3: Computed spin-polarized band structures at different hole densities

(a) $0.6 \times 10^{13} / \mathrm{cm}^{2}$, (b) $1.0 \times 10^{14} / \mathrm{cm}^{2}$, and (c) $1.7 \times 10^{14} / \mathrm{cm}^{2}$. Blue and black lines indicate spin-up like and spin-down like states, respectively. 


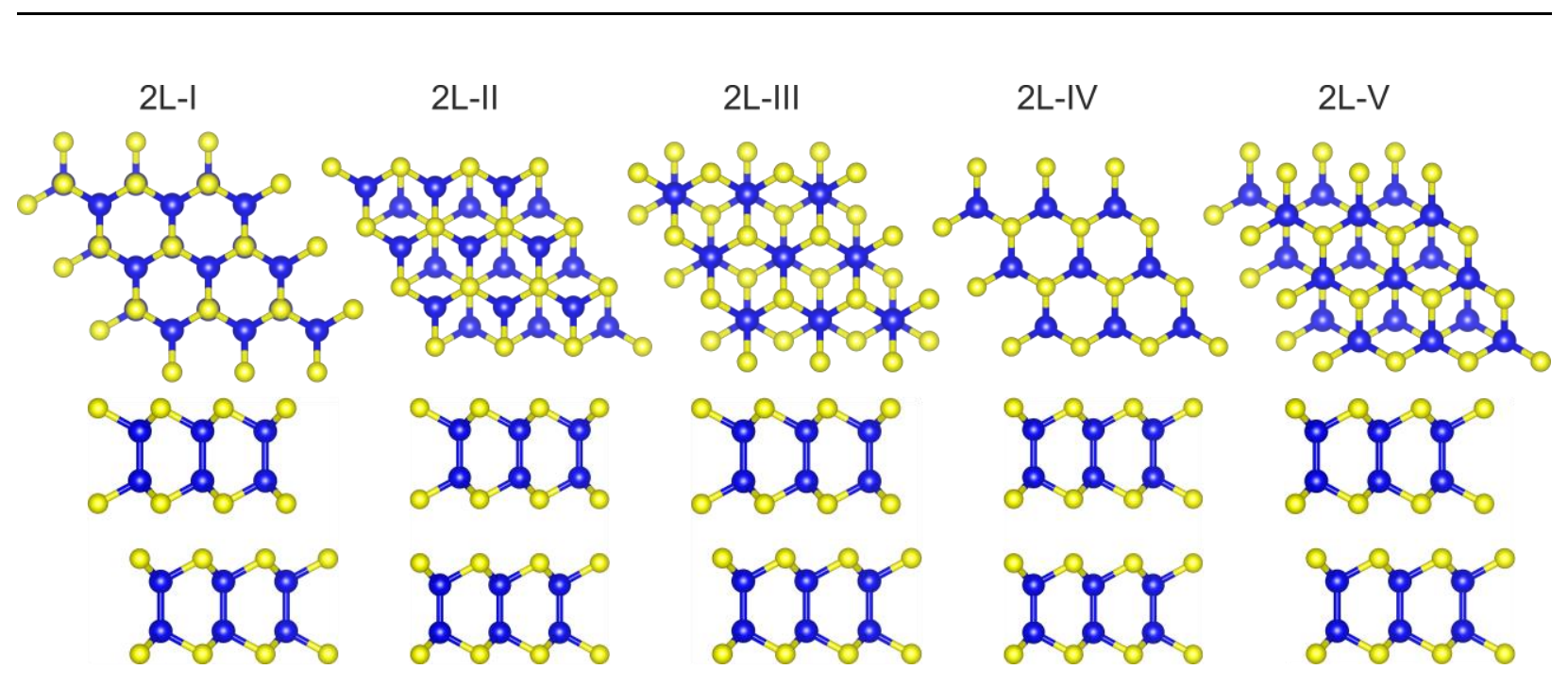

Figure 4: Top and side views of bilayer InSe with different stacking patterns. 


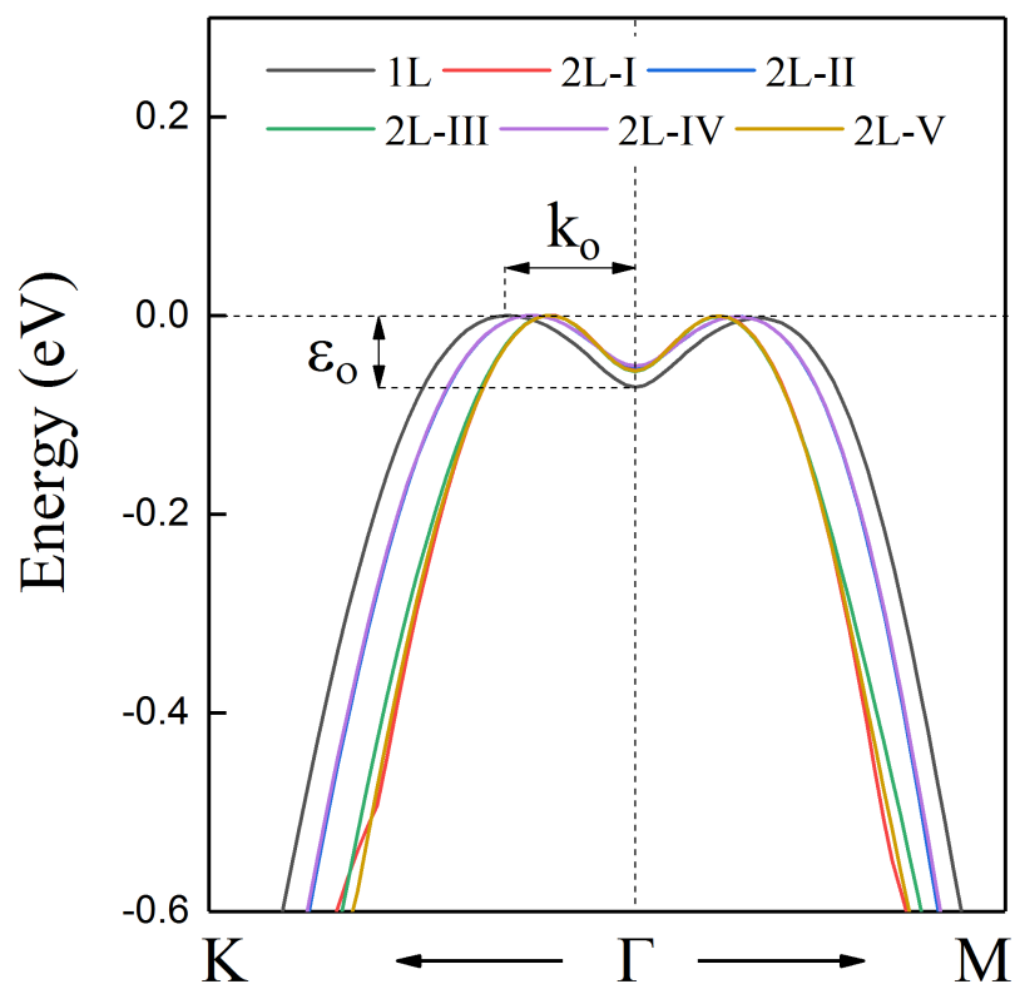

Figure 5: Topmost valence band of bilayer InSe with various stacking patterns. For all cases the valence band top is set at zero point. For the sake of comparison, the topmost valence band of monolayer InSe is also presented. 


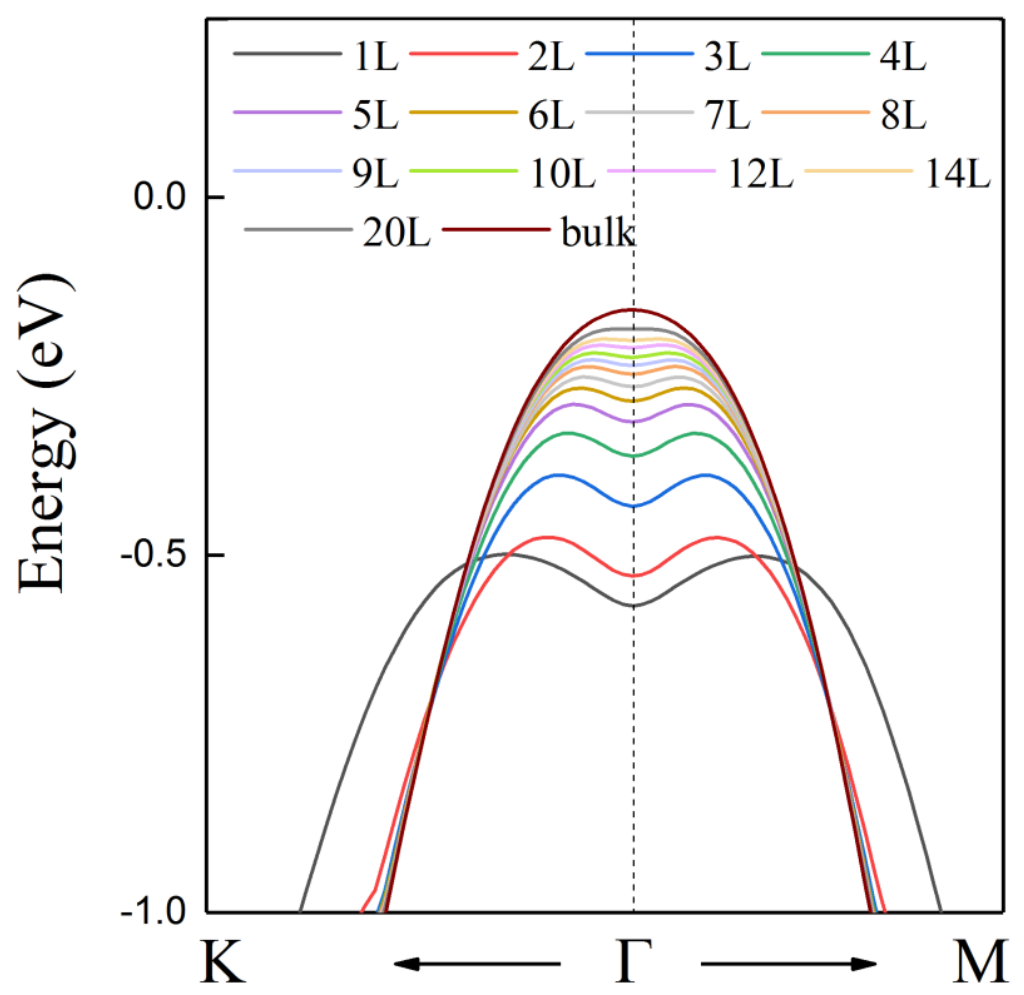

Figure 6: Evolution of the topmost valence band of $\beta$-InSe by increasing its thickness. 
(a)

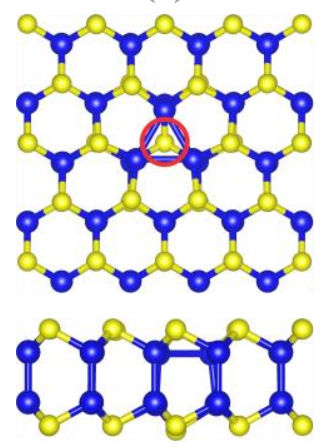

(d)

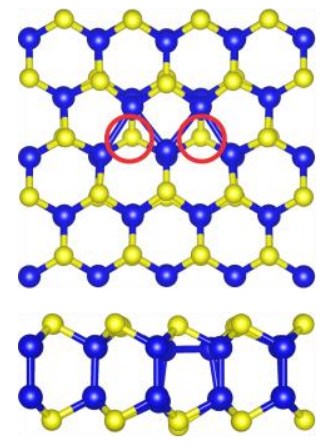

(g)

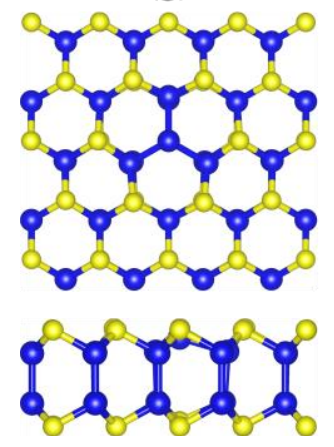

(b)

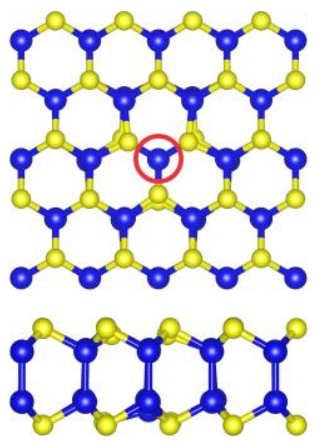

(e)
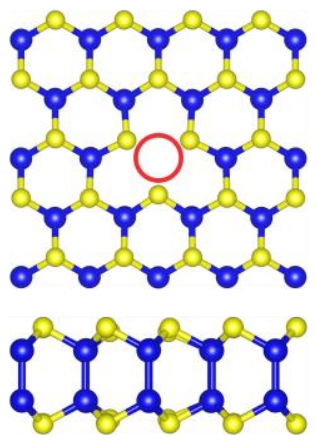

(c)

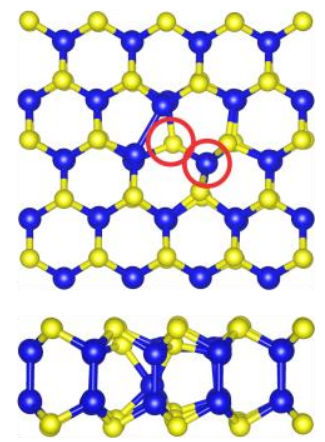

(f)

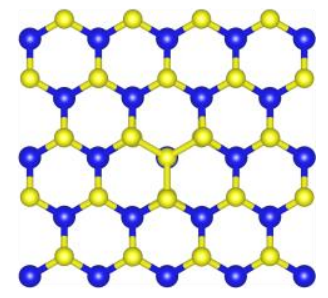

11:1:

Figure 7: Top and side views of the relaxed atomic structures of defective InSe monolayers (a) Se vacancy, (b) In vacancy, (c) Se-In di-vacancy, (d) Se di-vacancy, (e) In di-vacancy, (f) Se antisite, (g) In antsite. Blue and yellow spheres correspond to In and Se atoms, respectively. Red circles indicate the position of the vacant sites. 


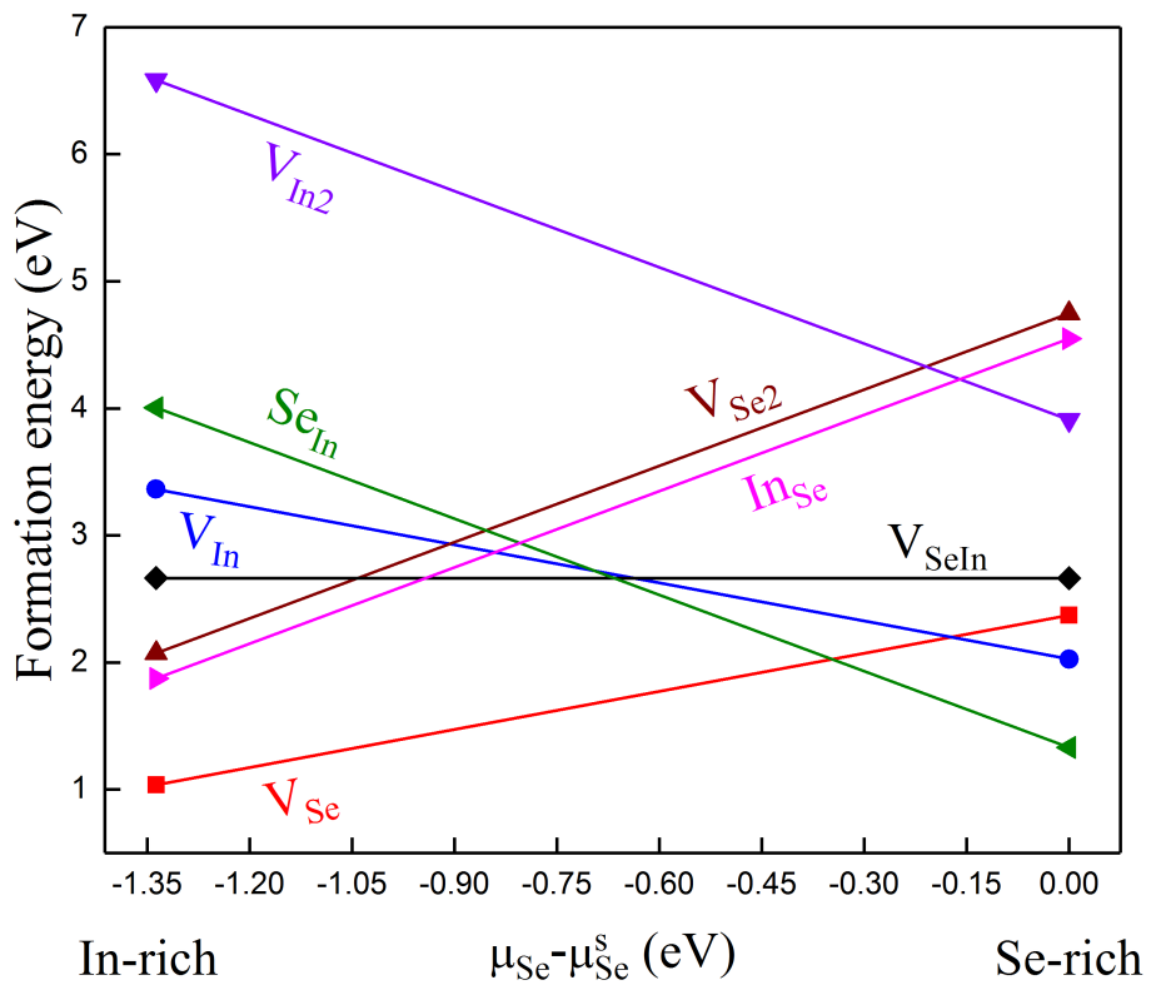

Figure 8: Defect formation energies of single-layer InSe, in a wide range of Se chemical potential values. 
(a)

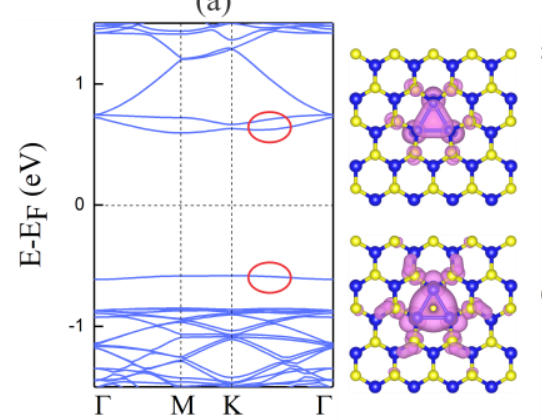

(d)

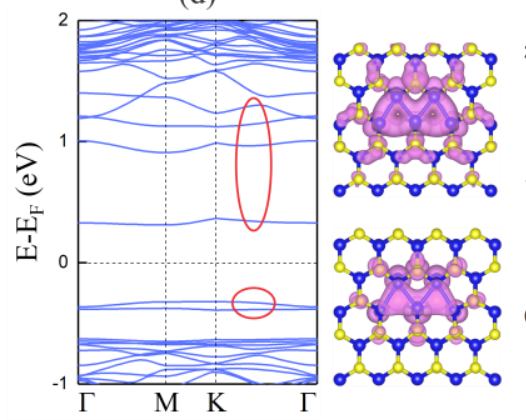

(g)

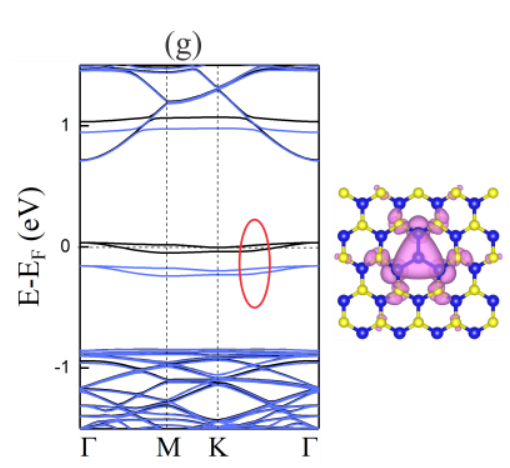

(b)

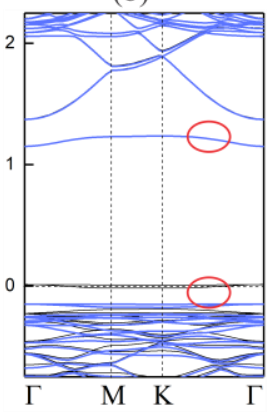

(e)

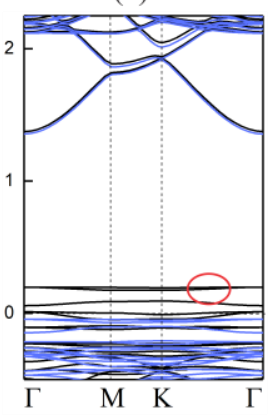

(c)

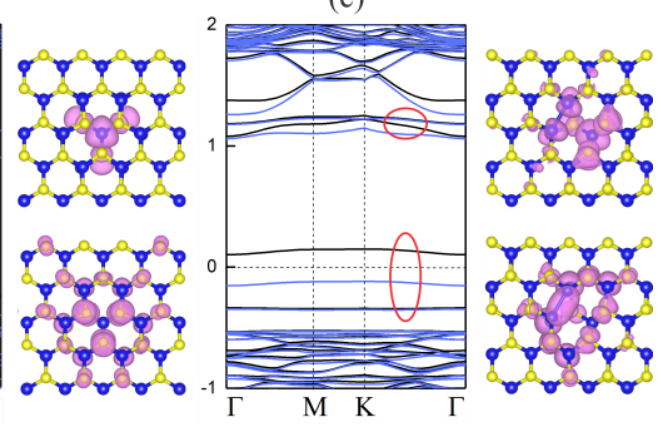

(f)

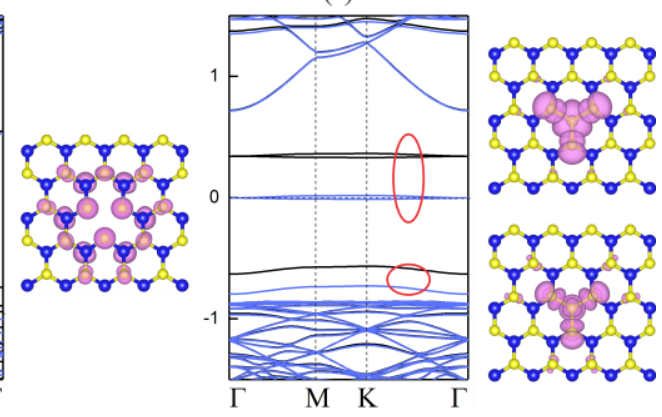

Figure 9: Computed band structures of InSe monolayers using the PBE functional and partial charge densities of defect-related states, which are highlighted by red circles. (a) Se vacancy, (b) In vacancy, (c) Se-In di-vacancy, (d) Se di-vacancy, (e) In di-vacancy (f) Se antisite, (g) In antisite. For the spin polarized band structures, blue and black lines indicate spin-up like and spin-down like states, respectively. For the charge densities, the isosurface value is 0.001 electrons $\AA^{-3}$. 
(a)

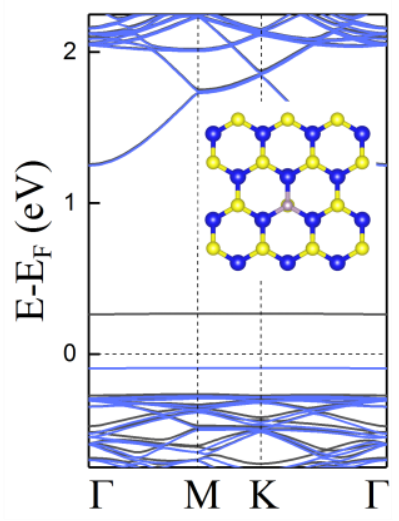

(e)

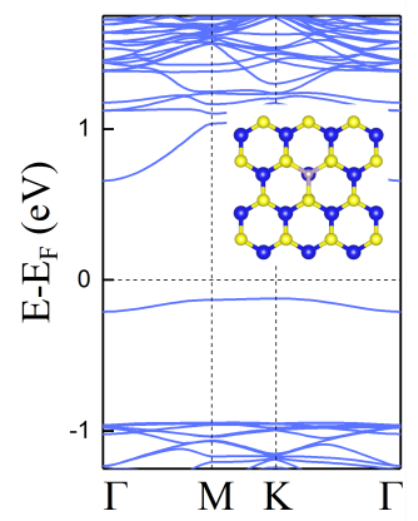

(b)

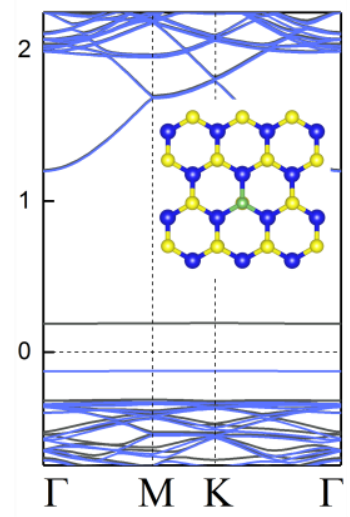

(f)

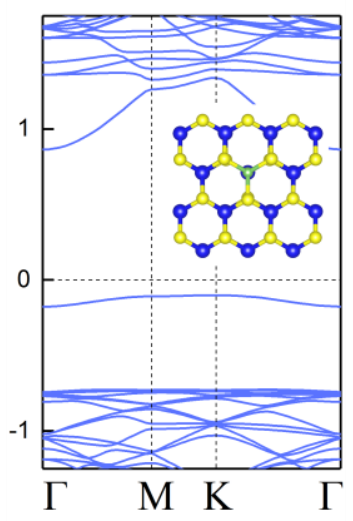

(c)

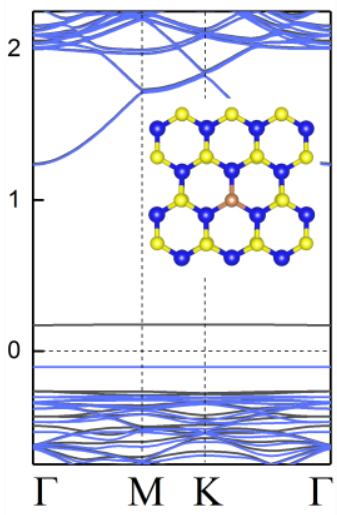

(g)

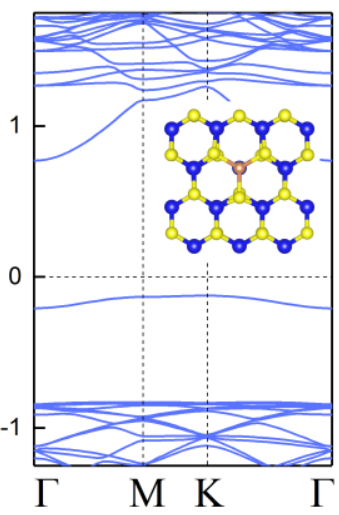

(d)

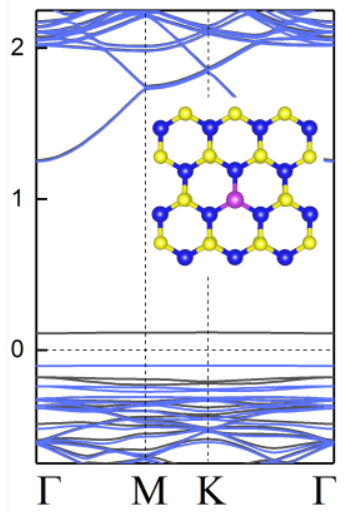

(h)

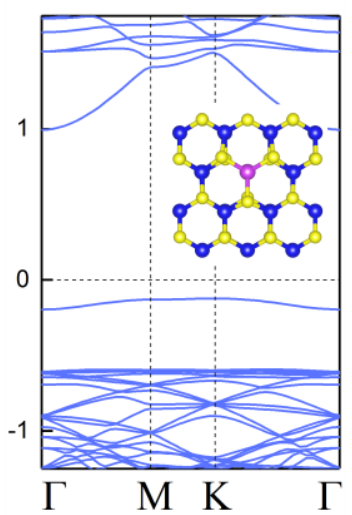

Figure 10: Relaxed atomic structures and computed band structures of InSe monolayers with group-V substitutional impurities. (a) $\mathrm{P}$ in place of $\mathrm{Se}$, (b) As in place of $\mathrm{Se}$, (c) $\mathrm{Sb}$ in place of $\mathrm{Se}$, (d) Bi in place of Se, (e) P in place of In, (f) As in place of In, (g) Sb in place of In, (h) Bi in place of In. Purple, green, orange, and pink spheres correspond to $\mathrm{P}, \mathrm{As}, \mathrm{Sb}$, and $\mathrm{Bi}$ atoms, respectively. For the spin polarized band structures, blue and black lines indicate spin up like and spin down like states, respectively. 


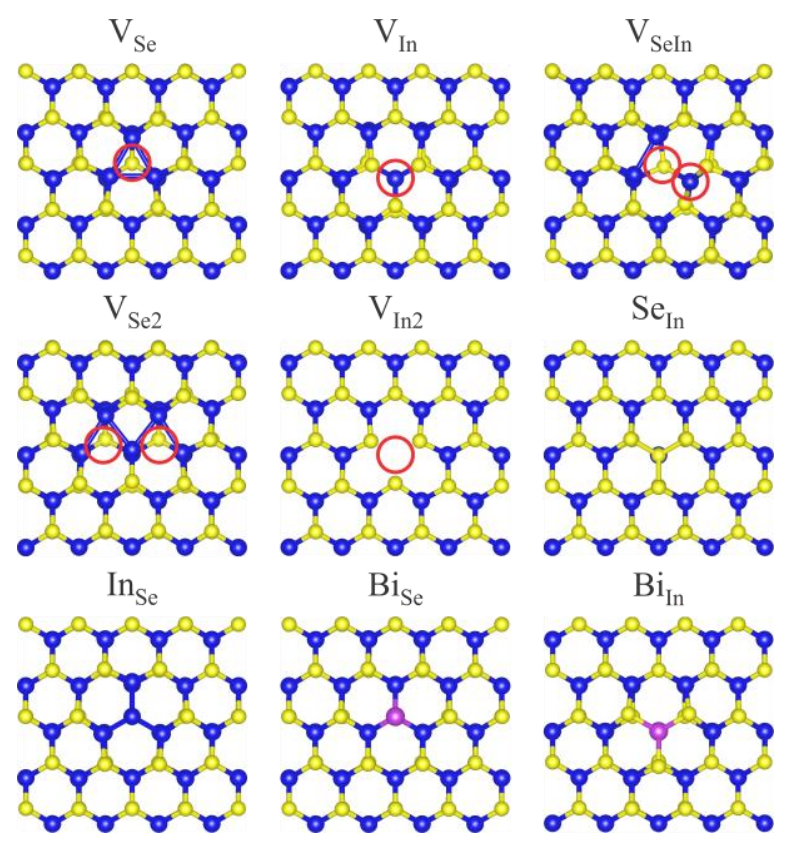

Graphical Abstract 